\title{
Bilateral anatomical variations of the hand extensors
} Ferreira Arquez, $H$

\begin{abstract}
:
Background: The purpose of this paper is to describe an anatomical variation of the hand extensor on the little finger of the right hand which receives four tendons, condition unknown, as it is the first case reported so far in the literature. The human extensor tendons of hand often display an array of variations. Awareness of the anatomy and variations of the extensor tendons on the dorsum of the hand is necessary not only for the anatomist but also for surgeons when considering tendons for hand surgery, tendon rerouting or transplants. Materials and Methods: Bilateral anatomical variation in the upper limb was found during routine dissection in a 75-year-old male cadaver in the Morphology Laboratory at the University of Pamplona. The variations were recorded and photographed. Result: In the left hand the extensor digitorum there was a single tendon to the index, double tendon to the middle, triple tendon to the ring finger, a single tendon to the little finger or digiti minimi. The extensor digiti minimi has double tendon. In the right hand the extensor digitorum there was a single tendon to the index, triple tendon to the middle finger, triple tendons to the ring finger, a double tendon to the little finger. The extensor digiti minimi has double tendon. The little finger receives four tendons, 2 of extensor digitorum and 2 of extensor digiti minimi. The dorsum of the left hand and right showed juncturae tendinum type 2, between the extensors tendons in the 3rd intermetacarpal space; type 3, between the extensors tendons in the 4th inter metacarpal space. Conclusion: The presence of multiple tendons may alter the kinematics around the site of attachment to the phalanx. Knowledge of anatomical variations and normal anatomy of the extensor tendons, may be helpful while performing graft and tendon transfer operations.
\end{abstract}

Keywords: anatomical variations; extensor digitorum muscle; extensor digiti minimi muscle; juncturae tendinum; extensor retinaculum; accessory tendons; dorsum; hand

Bangladesh Journal of Medical Science Vol. 15 No. 02 April'16. Page : 278-282

\section{Introduction}

The hand is one of the special organs of human body which is most frequently injured. The synergistic contraction of the extensor musculature along with the long flexors is mandatory for an efficient grip on different objects in daily life ${ }^{1}$. A detailed knowledge of the extensor tendons anatomy is essential for under standing the consequences of tendon injury at various levels. This tendon injury may be either due to external trauma or spontaneous rupture as in patients with rheumatoid arthritis and distal radio ulnar joint osteoarthritis ${ }^{2}$. Usually the dorsal surface of the hand is devoid of muscle bellies, it only contains the tendons of the extensor muscles of the hand. Between the dorsal carpal ligament and the carpal bones six compartments are formed for the passage of tendons. Found on the dorsum of the hand are the tendons of the extensor pollicis longus (EPL), extensor digitorum (ED), extensor indicis (EI), and the extensor digiti minimi (EDM). However, the existence of variant muscles on the dorsum of the hand has been documented in numerous publications ${ }^{3}$.Finger extension involves simultaneous actions of both extrinsic and intrinsic extensor muscles. Extrinsic muscles originate in the elbow and forearm and include the following: extensor digitorum, extensor indicis, and extensor digiti minimi. The extensor digitorum muscle originates to level lateral epicondyle of the humerus and ends in four tendons, one for each digit except for the thumb. The extensor digiti minimi muscle originates to level lateral epicondyle of the humerus and ends in one tendon that inserts into the extensor expansion of the 5 th finger.

Corresponds to: Humberto Ferreira Arquez, University of Cartagena. University St. Thomas. Professor Human Morphology, Medicine Program, University of Pamplona. Morphology Laboratory CoordinatorUniversity of Pamplona. E-mail: humfear@unipamplona.edu.co 
The primary function of these muscles is extension of the metacarpophalangeal (MCP) and interphalangeal joints. The intrinsic muscles that originate and insert within the hand are the lumbrical and interosseous muscles. The function of these muscles is primarily to extend the interphalangeal joints and secondarily to contribute to flexion of the metacarpophalangeal joint. These groups of extrinsic and intrinsic muscles are coordinated by a series of stabilizing retinacular structures, which facilitate balanced transmission of muscular force. These structures are found in the dorsum of the carpum is extensor retinaculum, the hands is intertendinous connections, and the fingers are extensor hood, retinacular and triangular ligaments $s^{4,5}$.

Connexus intertendineus, juncturae tendinum (JT) or oblique tendinous connections between the extensor tendons of the fingers on the dorsum of the hand are commonlyobserved.Three types of juncturae tendinum $(\mathrm{JT})$ are identified between the tendons of extensor digitorum in the 2nd, 3rd and 4th intermetacarpal spaces (IMS) of hands. Type 1 juncturae tendinum, made of thin filamentous intertendinous fascia; Type 2 juncturae tendinum, formed of dense thick well defined ligamentous bands; Type 3 juncturae tendinumwhich consisted of tendon slips, was the most frequently identified in all juncturae ${ }^{6}$. Anatomical variation of the extrinsic extensor tendons are frequent and knowledge is important when assessing the traumatized or diseased hand and provide significant information to hand surgerons, when considering tendons for repair or transfer.

\section{Materials and methods}

The described human extensor tendons anatomic variations were found in the right and left side of a male cadaver of 75 years of age during routine upper limbs dissection in the Morphology Laboratory at the University of Pamplona developed by training medical students. The history of the individual and the cause of death are not known. The topographic details were examined by casual dissection and the variations were recorded and photographed.

\section{Results}

Left hand: In the left forearm the extensor digiti minimi (EDM) muscle originates to level lateral epicondyle of the humerus, passing inferiorly to the extensor retinaculum (ER) ends in double tendons that inserts into the extensor expansion of the 5th finger. The extensor digitorum(ED) muscle originates to level lateral epicondyle of the humerus and continued downwards, passing inferiorly to the extensor retinaculum (ER) to split into individual tendons for each of the finger. There was a single tendon to the index, double tendon to the middle, triple tendon to the ring finger, a single tendon to the little finger. The dorsum of the hand showing juncturae tendineum type 2, between ED 3, ED 4 in the 3rd. intermetacarpal space of a left hand; juncturae tendineum type 3 between ED 6, ED 7 in the 4th intermetacarpal space of a left hand. The extensor digiti minimihas double tendon. The little finger receives three tendons.

Figure 1. Left Hand: EI: Extensor indicis tendon; E.D: Extensor digitorum; ED 1: single tendon of extensor digitorum to the index finger; ED 2, ED 3: double tendon of extensor digitorum to the middle finger; ED 4, ED 5, ED 6 : triple tendons of extensor digitorum to the ring finger; ED 7: tendon of extensor digitorum to the little finger; EDM: Extensor digiti minimi; EDM 1, EDM 2: double tendon inserted into little finger. The dorsum of the hand showing juncturae tendinum type 2, between ED 3, ED 4 in the 3rd. intermetacarpal space of a left hand; and juncturae tendinum type 3, between ED 6, ED 7 in the 4th intermetacarpal space of a left hand.

Right hand: In the right foream the extensor digiti minimi (EDM) muscle originates to level lateral epicondyle of the humerus, passing inferiorly to the extensor retinaculum (ER) ends in double tendons that inserts into the extensor expansion of the 5th finger. The extensor digitorum (ED) muscle originates to level lateral epicondyle of the humerus continued downwards, passing inferiorly to the extensor retinaculum to split into individual tendons for each of the finger. There was a single tendon to the index, triple tendon to the middle finger, triple tendons to the ring finger, a double tendon to the little finger. The dorsum of the hand showing juncturae tendinum type 2, between ED 3, ED 4, ED 5 in the 3rd. intermetacarpal space of a right hand; juncturae tendinumtype 3, between ED 7, ED 8 in the 4th intermetacarpal space of a right hand. The extensor digiti minimihas double tendon. The little finger receives four (4) tendons 2 of extensor digiti minimi and 2 of extensor digitorum.

Figure 2. Right hand. EI: Extensor indicis; ED: extensor digitorum; ED 1: single tendon to the index; ED 2,ED 3,ED 4: three tendons to the middle finger; ED 5, ED6, ED7: three tendons to the ring finger; $\mathrm{ED}$ 8, $\mathrm{ED}$ 9: double tendon inserted into little finger; EDM: extensor digiti minimi; EDM 1, EDM 2: double tendon inserted into little finger; The dorsum of the hand showing juncturae tendinum type 2, between ED 3, ED 4, 


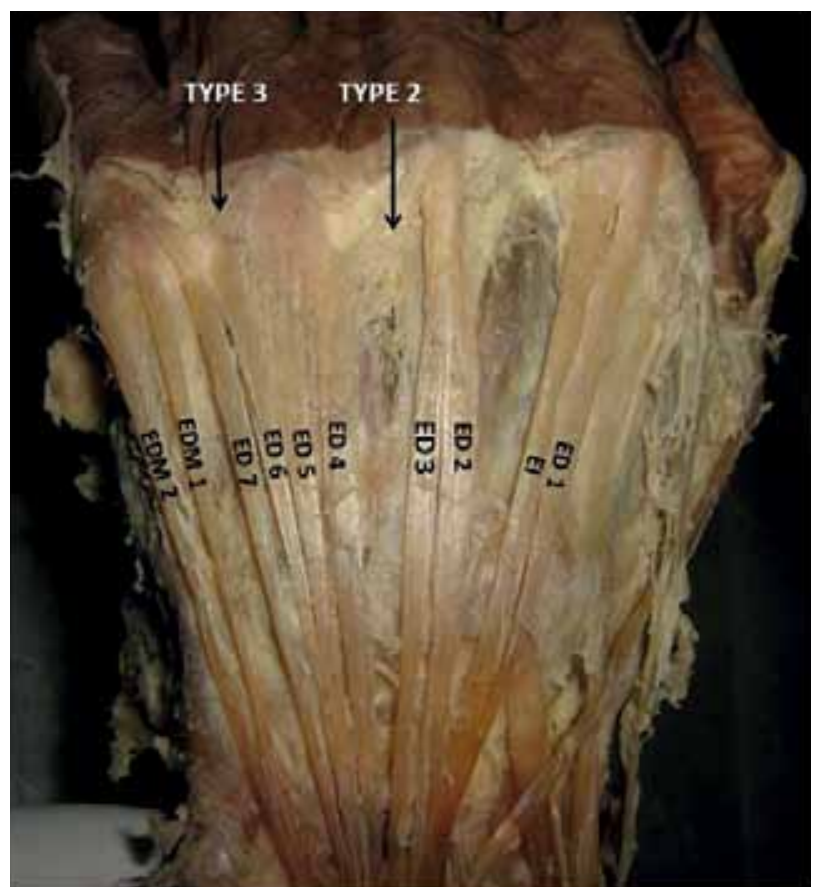

Figure 1

ED 5 in the 3rd. intermetacarpal space of a right hand; and juncturae tendinum type 3 , between ED 7 , ED 8 in the 4th intermetacarpal space of a right hand. The little finger receives four tendons, 2 of ED and 2 de EDM

\section{Discussions}

The tendon of the extensor digitorum may exhibit multiple variations in term of number. Even the standard textbooks of anatomy mention that the tendon ofextensor digitorum may be doubled or even tripled in any of the digits and such a variations has been reported to be more common in the index or the middle finger ${ }^{7,8}$. Hence, even a double tendon to any of the digits may be taken as usual findings. There are even reports of double or triple tendons to the long fingers, single or double tendons to the little finger and occasionally double tendons to the little finger ${ }^{9}$.

Nayak et al. (2008) remarks, on this purpose, that 'the extensor compartment of the forearm is one of the regions of the human body with frequent variations of its content' ${ }^{10}$.

Abdel-Hamid et al. (2013) report a single tendon of extensor indicis in all cases of the specimens studied $^{4}$. Dass et al. (2011) detected a single tendon ofextensor indicis in $98 \%$ of specimens ${ }^{11}$. In the current case as well as previous studies ${ }^{4,12,13}$, the extensor indicis tendon was always medial and deep to the tendon of extensor digitorum to the index finger. The extensor indicis permits independent extension of the index finger and is commonly used

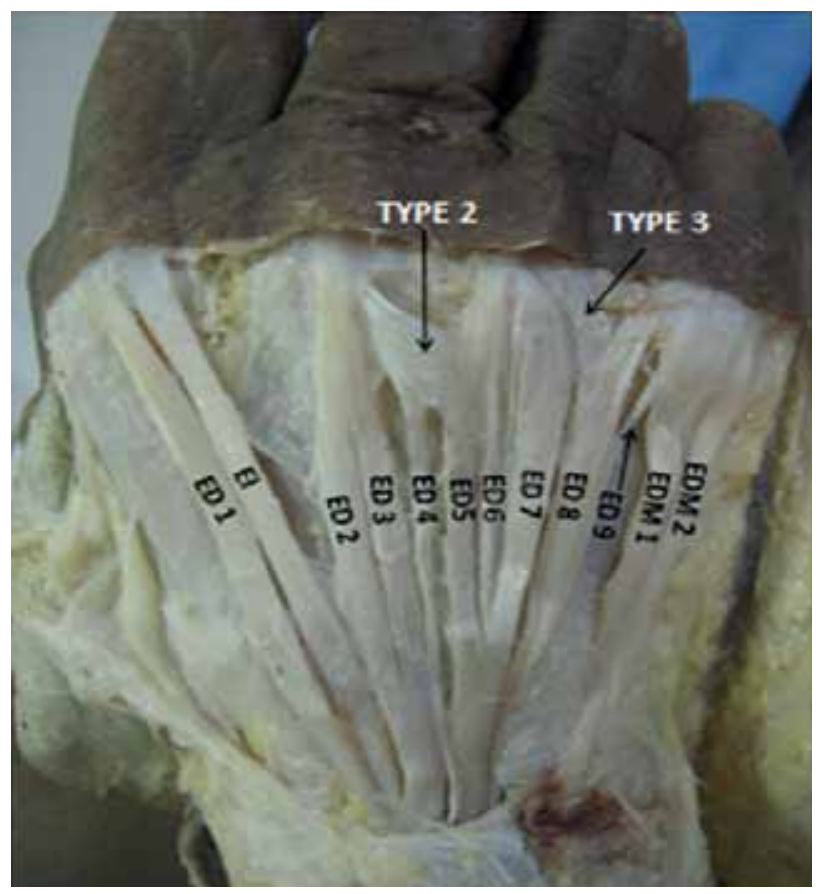

Figure 2

for tendon transfer ${ }^{14,15}$.

The number of extensor digitorum tendons may vary from 3 to 7 proximal to the extensor retinaculum and from 4 to 8 distal to it. Although, the incidence of 4 tendons $(32.6 \%)$ and 6 tendons $(40 \%)$ were the commonest both proximal and distal to the extensor retinaculum ${ }^{4}$. El-Badawi et al.(1995) reported 2-6 extensor digitorum tendons and 3-8 tendons proximal and distal to the ER respectively; 3 tendons $(55.2 \%)$ and 4 tendons $(60.77 \%)$ were the commonest ${ }^{12}$.

Hirai et al. (2001) declared the presence of single tendons of the extensor digitorum to the index finger in $92 \%$ of specimens studied ${ }^{16}$; nevertheless, they were documented in all dissected specimens ${ }^{11,12,13}$, Abdel-Hamid et al. (2013) report single tendons of extensor digitorum to the index finger in $96.8 \%$ of specimens and double tendons only in $3.2 \%$ of cases ${ }^{4}$, in accordance to Von Schroeder et al. (1995) who detected them in $2 \%{ }^{9}$.

Single tendons of extensor digitorum to the middle finger was reported by Abdel-Hamid et al. (2013) in $(41,1 \%)^{4}$. They were also recorded in $51-64 \%$ of specimens in previous studies $9,12,13,16$. Single tendons of extensor digitorum to the ring finger were found in $(6.3 \%)^{4}$. Also von Schroeder et al(1995) and Zilber et al. (2004) reported single tendons for extensor digitorum to the ring finger in $12 \%$ and $18 \%$ respectively ${ }^{9,13}$. Contrary, much higher incidence of single tendon of extensor digitorum to the ring finger (62-96\%)observed by others studies 
$12,17,{ }^{18}$. Double tendons of extensor digitorum to the middle finger and extensor digitorum to the ring finger were observed in $46.3 \%$ and $36.8 \%$, respectively ${ }^{4}$. However, a variable incidence was recorded for their duplication, as low as $4 \%$ and $2 \%$ for extensor digitorum to the middle finger and extensor digitorum to the ring finger, respectively ${ }^{18}$ or as high as $39 \%$ for extensor digitorum to the middle finger ${ }^{16}$ and $63 \%$ for extensor digitorum to the ring finger ${ }^{9}$. Also, triple tendons of extensor digitorum to the middle finger $(12.6 \%)$ and extensor digitorum to the ring finger $(50.5 \%)$ was reported ${ }^{4}$.Nevertheless, this incidence was variable in previous studies ranging between 2 and 19\% forextensor digitorum to the middle finger ${ }^{11,12}$ and between 1 and $22 \%$ for extensor digitorum to the ring finger ${ }^{12,13}$. The percentage of quadruple tendons of extensor digitorum to the ring finger (6.3\%) reported by Abdel-Hamid et al. (2013) was on average between that documented by Hirai et al. (2001)of $4 \%$ and by Zilber and Oberlin (2004) of $8 \%$; though their existence could not be verified by some studies ${ }^{11,12,18}$. The tendon of extensor digitorum to the little finger was reported absent $t^{4}$. Yet, it was either replaced by a slip from the common bifurcating tendon to both ring and little fingers or by intertendinous connection with the ring finger. Similarly, the common bifurcating tendon and intertendinous connection were detected in $126 / 181(69.61 \%)$ of studied specimens ${ }^{12}$.

Other researchers recorded different percentages of absent extensor digitorum to the little finger ranging between $(2 \%)^{18}$ and $(66 \%)^{11}$. In specimens studied by Abdel-Hamid et al. (2013) where extensor digitorum to the little finger was absent, extensor digiti minimi gave 2 to 3 tendons to the little finger to replace its absence. This was supported by Dass et al. (2011)who suggested that in case of absent extensor digitorum to the little finger or presence of a common tendon dividing for both ring and little fingers, the extensor digiti minimi compensated by giving 2 or 3 tendons to the little finger ${ }^{11}$. A single tendon of extensor digiti minimi was found in all specimens proximal to the extensor retinaculum ${ }^{4}$. In accordance, single tendonof extensor digiti minimi was previously documented proximal to the extensor retinaculum in $(92-95 \%)^{11,12}$. Double tendons of extensor digiti minimi were detected in $75.8 \%$ and triple tendons in $15.8 \%$ distal to the extensor retinaculum (4). However, this duplication was recorded in (82$87 \%)^{9,11,16}{ }^{18}$ and triplication in $(2-8 \%)^{13,18}$. In the present case the little finger receives four tendons, 2 of extensor digiti minimi and 2 of extensor digitorum, this variations described is unique, has not been reported.

Reports often refer the presence of tendinous, oblique tendinous or intertendinous connections (juncturae tendinum) slips between the extensor tendons on the dorsum of the hand. Von Schroeder et al. (1995) classify these Juncturae Tendinum(JT) of the extensor tendons into three types: Type I juncturae consist of filamentous regions within the intertendinous fascia; Type II consists of much thicker and well-defined connecting bands; and Type III consist of tendon slips from the extensor tendons and were subclassified into «y» or « $\mathrm{r} »$ subtypes, depending on shape ${ }^{6}$. Hirai et al. (2001) in their series of 548 cadaveric hand dissections found that the most common pattern of intertendinous connections wereclassified into Type I in the second intermetacarpal space, into Type III-r in the third metacarpal space, and into type III-y in the fourth intermetacarpal space ${ }^{16}$. The functional importance of these juncturaewould be to strengthen the frail tendons of the extensor mechanism of the fingers, at the cost of some loss of independence of the 4th finger $^{9,20-23}$. Accordance with Gövsa et al. (2011) who described the $4^{\text {thintermetacarpal }}$ spacesto contain the thickest type of Juncturae Tendinum (JT). This type can be used for repairing lacerated or torn tendons, this suggestion was supported by the histological similarity between type 3 Juncturae Tendinum and tendons ${ }^{17}$.

Injuries to the extensor tendons of the fingers are common because of their poorly protected anatomic location. Clinical reports emphasize the importance of initial treatment and postoperative rehabilitation in achieving a good outcome ${ }^{24}$. Because the excursion of the extensor tendons over the fingers is less than that of the flexors, preservation of their length is far more critical to restoring the normal tendon balance ${ }^{25}$. The relationship between the location of injury and the outcome is an important concept. The type of injury, deformity, and surgical outcome will be different according to the affected anatomic regions. As a result, the categorical classification of tendon injuries into anatomic zones is crucial to the diagnostic process. As already mentioned, Verdan's zone system is the most widely accepted ${ }^{5,26}$.

\section{Conclusion}

Even though the extensor digitorum and extensor digiti minimi are asymptomatic in most cases, 
authors feel it is still essential for surgeons to be aware of possible variations of the extensor tendons and to ensure these variations are not overlooked in the differential diagnosis. These variations may be asymptomatic but when requiring surgical interventions an extra care must be taken especially during routine hand surgeries. Presence of these types of variant tendons on the dorsum of the hand in persons who are involved in sports such as, golf, cricket, tennis, weight lifting were the excessive wrist movement is involved, may show some symptoms. The presence of such thin tendons may be vulnerable to rupture in diseases like rheumatoid arthritis. If there is detailed knowledge of the anatomy and prevalence of this variations, it can help to prevent diagnostic errors, influence surgical and interventional procedures and avoid surgical complications during hand surgery.

\section{Acknowledgements}

The author, on behalf of the University of Pamplona thanked to the Erasmo Meoz University Hospital for the donation of cadavers identified, unclaimed by any family, or persons responsible for their care, process subject to compliance with the legal regulations in force in the Republic of Colombia.

Conflict of interest: None declared

\section{References}

1. Sinnatamby CS (2006) Last's anatomy: regional and applied. Churchill Livingstone, Edinburgh, pp. 76-80.

2. Casal D, Pais D, Bilhim T, Ribeiro V, Cunha S, Damásio C,Fernandes R, Angélica-Almeida M, Goyri-O’Neill J. A rare variation of the extensor indicis proprius tendon with important clinical implications. J Morphol Sci, 2011; 28: 208-211.

3. Singh K, Dennie D, Rao SR. Variant extensor muscles on the dorsum of the hand. Int J Anat Var (IJAV). 2009; 2: 80-82.

4. Abdel-Hamid GA, El-Beshbishy RA, Abdel Aal IH. Anatomical variations of the hand extensors. Folia Morphol (Warsz). 2013 Aug;72(3):249-57.

5. Clavero JA, Golano P, Farinas O, Alomar X, Monill JM,Esplugas $M$ Extensor mechanism of the fingers: MR imaging-anatomic correlation. Radiographics, 2003;23:593-611.

7. Williams PL et al. Gray's Anatomy. The Anatomical Basis of Medicine and Surgery. 38 ${ }^{\text {th }}$ Edition. Edinburgh, Churchill Livingstone 1997, p. 849-850.

8. Sinnatamby CS. Last's Anatomy. Regional and Applied. $10^{\text {th }}$ Edition. Edinburgh, Churchill Livingstone, 2001, p. 71-72.

9. Von Schroeder HP, Botte MJ. Anatomy of the Extensor Tendons of the Fingers: Variations and multiplicity. $J$ Hand Surg Amer 1995; 20 (1): 27-34.

10. Nayak SR, Krishnamurthy A, Prabhu LV, Rai R, Ranade AV, Madhyastha S. Anatomical variation of radial wrist extensor muscles: a study in cadavers. Clinics. 2008;63:85-90.

11. Dass P, Prabhu LV, Pai MM, Nayak V, Kumar G, Janardhanan JP. A comprehensive study of the extensor tendons to the medial four digits of the hand. Chang Gung Med J. 2011; 34: 612-619.

12. El-Badawi MG, Butt MM, Al-zuhair AGH, Fadel RA. Extensor tendons of the fingers; arrangement and variations: II. Clin Anat. 1995; 8: 391-398.

13. Zilber S, Oberlin C. Anatomical variations of the tendons to the fingers over the dorsum of the hand; a study of 50 hands and a review of the literature. Plast Reconstr Surg. 2004; 113: 214-21.
14. Patel MR, Moradia VJ, Bassini L, Lei B. Extensor indicis proprius syndrome: a case report. J Hand Surg Am. 1996; 21: 914-915.

15. Reeder CA, Pandeya NK. Extensor indicis proprius syndrome secondary to an anomalous extensor indicis proprius muscle belly. J Am Osteopath Assoc.1991;91: 251-253.

16. Hirai Y, Yoshida K, Yamanaka K, Inoue A, Yamaki K, Yoshizuka M. An anatomic study of the extensor tendons of the human hand. J Hand Surg Am. 2001;26:1009-15.

17. Gövsa F, Pinar Y, Çelik S, Bilge O, Sezak M. Anatomical similarity between tendons and Type 3 intertendinous connections: suitability as local donor tissue. Acta Orthop Traumatol Turc. 2011; 45: 370-375.

18. Godwin Y, Ellis H. Distribution of the extensor tendons on the dorsum of the hand. Clin Anat. 1992; 5: 394-403.

19. Joshi SS, Joshi SD. Applied significance of variations of the first extensor compartment of wrist. J Anat Soc India. 2002; 51: 159-161.

20. Von Schroeder HP, Botte MJ. Functional anatomy of the extensor tendons of the digits. Hand Clin. 1997;13:51-62.

21. Nimbarte AD, Kaz R, Li ZM. Finger joint motion generated by individual extrinsic muscles: a cadaveric study. J Orthop Surg Res. 2008;3: 27.

22. Klena JC, Riehlt JT, Beck JD. Anomalous extensor tendons to the long finger: a cadaveric study of incidence. $J$ Hand Surg Am. 2012;37:938- 41.

23. Pinar Y, Gövsa F, Bilge O, Celik S. Accessory tendon slip arising from the extensor carpi ulnaris and its importance for wrist pain. Acta Orthop Traumatol Turc. 2012;46:132-5.

24. Blair WF, Steyers CM. Extensor tendon injuries. Orthop Clin North Am 1992; 23:141-148.

25. Minamikawa Y, Peimer CA, Yamaguchi T, et al. Wrist position and extensor tendon amplitude following repair. J Hand Surg [Am] 1992; 17:268-271.

26. Kleinert HE, Verdan C. Report of the Committee on Tendon Injuries. J Hand Surg [Am] 1983; 8:794-798. 